\title{
What is the role of computational models in Cognitive Science? A quantitative and qualitative analysis of the history of the TRACE model of speech segmentation*
}

\author{
M. Chawla and R.C.Shillcock ${ }^{\dagger}$ \\ School of Informatics, The University of Edinburgh, \\ Edinburgh, UK \\ †-mail:rcs@inf.ed.ac.uk
}

\begin{abstract}
Implemented computational models are a central paradigm of Cognitive Science. How do cognitive scientists really use such models? We take the example of one of the most successful and influential cognitive models, TRACE (McClelland \& Elman, 1986), and we map its impact on the field in terms of published, electronically available documents that cite the original TRACE paper over a period of 25 years since its publication. We draw conclusions about the general status of computational cognitive modelling and make critical suggestions regarding the nature of abstraction in computational modelling.
\end{abstract}

Keywords: TRACE model; Speech segmentation; Computational modelling; Abstraction; Idealization .

\section{Introduction}

The computational metaphor has increasingly defined Cognitive Science since it emerged as a distinct discipline from the Cognitive Revolution of the 1950s. Cognition is standardly seen in terms of a self-contained individual mediating perceptual or linguistic "inputs" and linguistic or behavioural "outputs". The mediation, usually seen as carried out inside a single nervous system, is generally understood in terms of computations carried out over representations of different kinds.

A major qualitative advance came about when researchers went beyond the simple brain-as-a-computer linguistic metaphor and began writing programs that carried out computations that could be compared with the operations by which cognition was defined. Thus, if a computer program could independently generate, for instance, the past tense of a verb (e.g., talk $\rightarrow$ talked, catch $\rightarrow$ caught) then it was seen as a model or theory of how humans carried out the same process. "Model" and "theory" have been used broadly interchangeably.

Computational modelling is now a central paradigm in cognitive psychology and in cognitive neuroscience. Computational Linguistics is an independent discipline and other computational paradigms have leached back

\footnotetext{
* The citation analysis was carried out by the first author in completion of the MSc in Cognitive Science and Natural Language Processing, University of Edinburgh ${ }^{1}$. See [1] for complete details.
} 
into the subdomains of formal linguistics. Modelling has attracted increasing attention from philosophers. Such has been the technical and academic development in all these areas, Cognitive Science is now itself perhaps best defined by the implemented cognitive model, the most salient aspect of the computational metaphor.

Most researchers involved with computational cognitive modelling would point to the following advantages of the paradigm. (a) Models are empirical research: they reveal complex emergent behaviours, and patterns of behaviour, that could not be fully anticipated without the independent activity of the implemented model. (b) Models can help elaborate a conceptual vocabulary for the processing domain under study. (c) Models make predictions that can be tested with human subjects, in a "virtuous spiral" of investigation. (d) A working model contributes to - or even constitutes - an explanation of the observed human behaviours. (e) Cognitive modelling is a scientific pursuit: more is found out about the processing domain under study and it is situated within the rest of what we know about cognition. (f) Scientific understanding is advanced by comparing models in their ability to account for critical or benchmark data.

A glance at the philosophical distinctions made in the discussion of models in science ${ }^{2}$ reveals even greater complexity immanent in this issue. Below, we contribute to this debate by the quantitative and qualitative analysis of a quarter of a century in the life of a premier computational cognitive model.

We ask, what is the material reality of what cognitive scientists actually do with computational cognitive models?

\section{The TRACE Model}

McClelland and Rumelhart had extensively explored the principles of the interactive-activation architecture in a model of letter perception ${ }^{3,4}$, and with the TRACE model ${ }^{5} \mathrm{McClelland}$ and Elman applied a temporal version of that architecture to the domain of speech perception.

The original hand-wired model contains three levels: phonemic features (7); phonemes (14); words (211). Input is to the first level. Any feature can increase the activation level of the phonemes in which it occurs; any phoneme can increase the activation level of the words in which it occurs. Conversely, there is "top-down" activation of parts by wholes. Activation is constrained by within-level inhibition as incompatible entities compete. In each level, multiple representations of the same entity are spread across a temporal dimension consisting of discrete time-slots, approximating graded activation across time. Parameters are set for excitation between adjacent levels, and for inhibition and decay within a level. The output is a "trace" of the activation across time of all of the model's constituents, which indicates psychological accessibility. In 2007, 
Strauss et al. introduced jTRACE a Java-based reimplementation and extension of the original C-based model ${ }^{6}$.

\section{Our methodology}

We analysed the 25-year citation history of the original (1986) TRACE publication as represented in Web of Science (WoS) and Google Scholar to yield respectively a descriptive analysis (types of document, most frequently cited articles, authors and their demographics) and a content analysis (a typology of the ways of referring to TRACE) based on the electronically available documents (as of 11/8/2011). (WoS provides more statistical citation data; Google Scholar provides links to many more (1286 versus 774 ) documents.)

\section{Descriptive Analysis}

WoS identified 774 documents citing the 1986 paper: 666 journal articles, with proceedings papers and "reviews/overviews" accounting for most of the rest. The authors chiefly hailed from US (437), UK (131), Netherlands (125), France, Germany, Canada, Belgium, Switzerland, Spain, Australia and Japan, and their combinations. Of the 277 authors, the five most frequently cited were (in order): (1) Plaut, D.C., McClelland, J.L., Seidenberg, M.S., \& Patterson K. (1996). Understanding normal and impaired word reading: Computational principles in quasi-regular domains. Psychological Review, 103(1), 56-115.

(2) Grainger, J. \& Jacobs, A.M. (1996). Orthographic processing in visual word recognition: A multiple read-out model. Psychological Review, 103(3), 518-565. (3) Luce, P.A. \& Pisoni, D.B. (1998). Recognizing spoken words: The neighborhood activation model. Ear and Hearing, 19(1), 1- 36.

(4) Hickok, G. \& Poeppel, D. (2007). The cortical organization of speech processing. Nature Reviews Neuroscience, 8, 393-402.

(5) Usher M. \& McClelland, J.L. (2001). The time course of perceptual choice: The leaky, competing accumulator model. Psychological Review, 108(3), 550-592.

A picture emerges of a large research community, with publications heavily skewed to a small percentage of particular North American and West European researchers. Fig. 1 shows yearly citations of the 1986 TRACE paper; when plotted cumulatively (not shown), WoS citations of the paper produce a curve only slightly less steep than that for references to "speech perception models". 


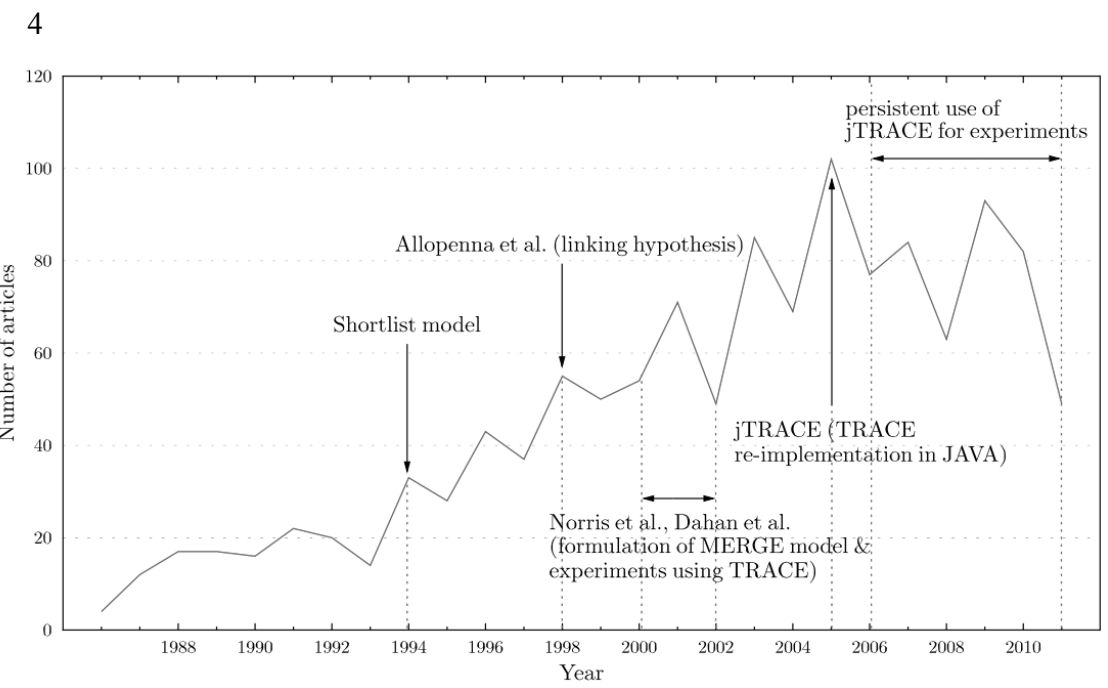

Fig. 1. Research involving TRACE, 1986 to 2011, as it affected citation of the 1986 paper.

\section{Content Analysis}

We individually examined the 776 open access papers (arguably the most influential citations) and iteratively categorized the contexts in which TRACE was cited, producing five categories (Fig. 2):

(i) TRACE simulations (Fig. 3). Like all except the brief mentions, these papers are a small proportion of the papers citing TRACE. They may describe parametric manipulations of the original model (often to contest architectural principles such as feedback between levels) or minor scaling-up of the lexicon. They often involve computational experiments that explore particular configurations of parallel activation of words or phonemes, and which may be compared with human behaviours such as eye-movements. McClelland (1991) enhanced TRACE by incorporating random noise and a discrete decision rule, to account for context effects. Mirman et al. (2008) ${ }^{7}$ reported two ways, within the existing architecture, of modulating the activity of the lexical level to simulate effects of attention. Finally, a special case is Strauss et al.' $\mathrm{s}^{6}$ (2007) faithful reimplementation (and release) of TRACE in Java as jTRACE, replicating a number of early studies. Some 16 articles cited jTRACE between 2005 and 2011.

(ii) Proposed new models of speech perception. These papers describe new models and contrasted them with TRACE. The major models are listed below: 


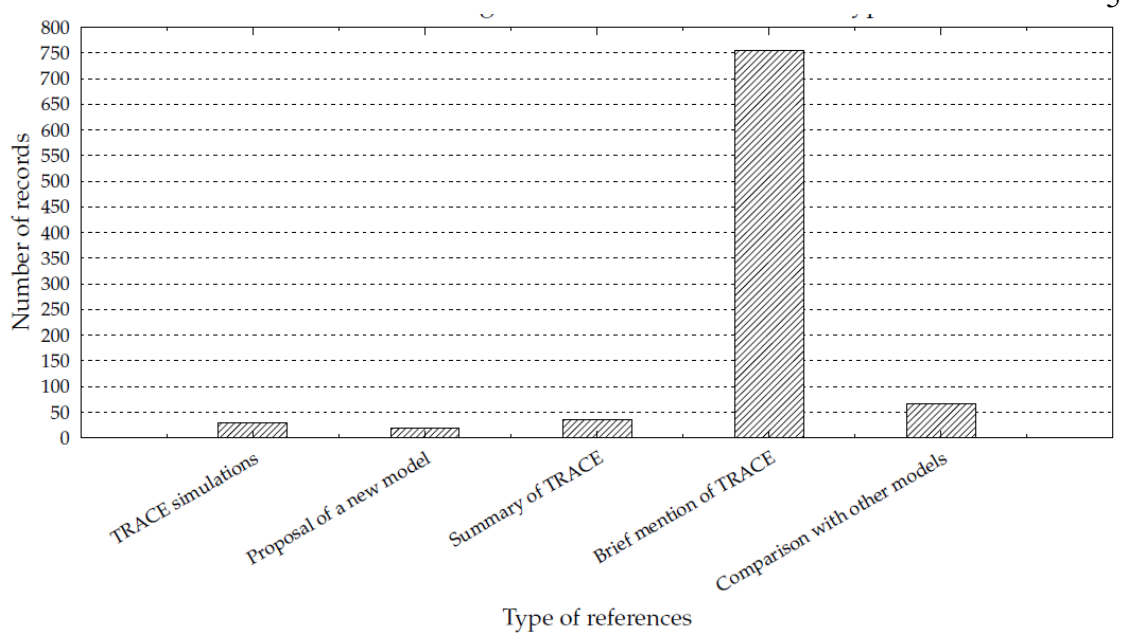

Fig. 2. Articles referring to TRACE divided into five types.

Shortlist ${ }^{8}$ employs a simulated recurrent neural network in conjunction with a limited lexical competition network working on the same principles as TRACE. PARSER $^{9}$ simulates segmenting words from continuous sentences that lack prosodic cues. The Neighborhood Activation Model (NAM) ${ }^{10}$ simulates the effects of neighbourhood density on speech perception, based on acousticphonetic patterns. The authors say "The NAM may, therefore, turn out to be virtually indistinguishable from an interactive-activation model". Luce et al. also present a connectionist version, PARSYN of the $\mathrm{NAM}^{11}$. The Distributed Cohort Model (DCM) ${ }^{12}$ (Gaskell \& Marslen-Wilson, 1997), like TRACE, was inspired by the original, non-implemented Cohort Model. The DCM employs a recurrent network, taking as input successive feature-level descriptions and generating at output separate "semantic" and phonological representations corresponding to the whole word. "Lexical" competition occurs between multiple distributed representations acquired by the hidden units during training. MERGE ${ }^{13}$ was an expansion of the Shortlist model that predicted the effect of lexical activation on phoneme identification as purely feedforward as opposed to the interactiveactivation framework assumptions. The authors compared a TRACE-like "interactive model" with their own MERGE model of phoneme perception. Shortlist $\mathrm{B}^{14}$ is a Bayesian alternative to the interactive-activation processing of the original Shortlist. The Fuzzy Logic Model of Perception (FLMP) ${ }^{15}$. TRACE and FLMP follow completely different sets of assumptions but the predictions made by both the models are quite similar. 


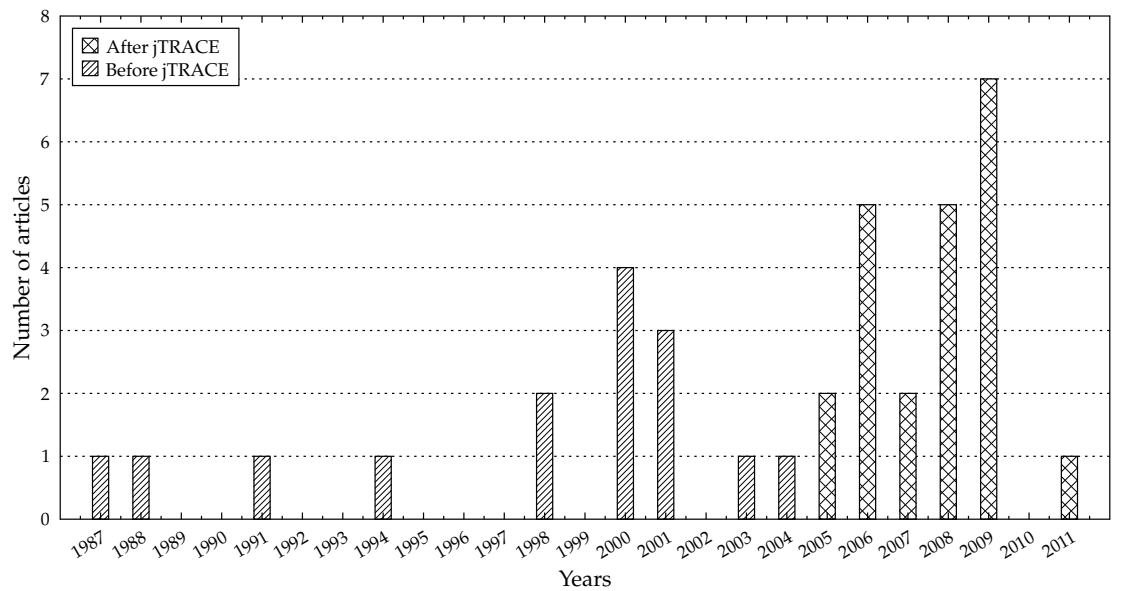

Fig. 3. Papers that report simulations with TRACE.

(iii) Papers that summarize TRACE briefly. A small number of papers provide summaries of TRACE as introductions to the issues of the modelling of speech perception, illustrating TRACE's role in the field.

(iv) Brief mentions. TRACE has been most widely cited in the form of brief references to the model; TRACE, and the theorizing behind it, is a landmark model in this sense. Brief mentions fell into five clear categories (Fig. 4):

(a) Properties of the model; e.g. TRACE's emergent segmentation behaviour, the type of connectivity, the nature of the input representation. Fig. 5 shows how the most frequently cited properties changed over time, reflecting shifting research emphases: there has been lasting interest in the processes of competition and activation. From 2001 there was sustained interest in the use of a pre-lexical level, reflecting the debate over bottomup versus top-down interaction.

(b) Debates about speech perception; e.g. should models be constrained to feedforward flow of information? Should lexical representations be allowed to affect phonetic representations? Connectionist models of language processing initially tended to have feedforward architectures trained by back-propagation, but were later augmented by recurrent connectivity. Phenomena such as phoneme restoration raised the issue of top-down processing. 


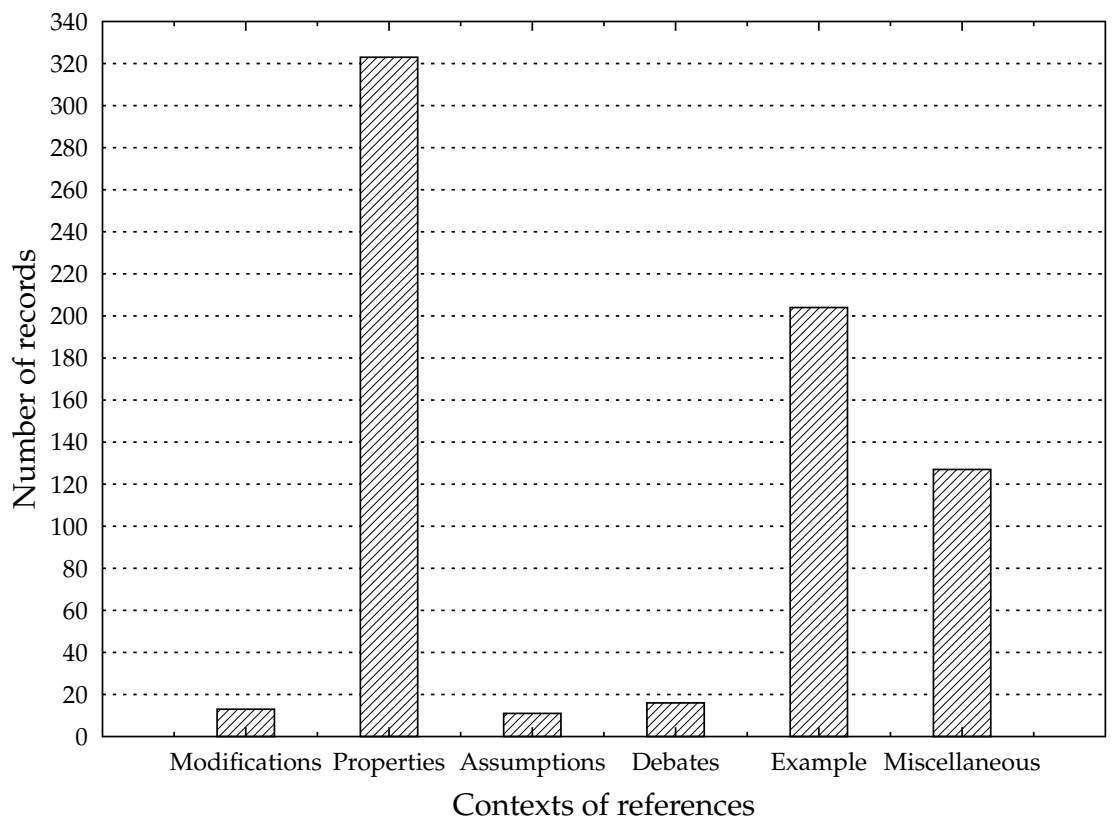

Fig. 4. Profile of the ways in which TRACE is referred to in the category of brief mentions.

(c) Assumptions concerning the model; e.g. feedback is assumed between different levels of representation; lexical representation is assumed to be phonetic, so that spoken input automatically activates lexical candidates in a continuous manner, without the necessary intervention of such representations as mora, syllables or stress units; TRACE incorporates some of the basic assumptions of the Logogen Model (Morton, 1969), with regard to units for words accruing activation.

(d) Modifications (suggested, but not implemented) to TRACE; e.g. could linguistic tone or visual features of the language be added to the model? Could TRACE represent morphemes in complex words? These proposed modifications keep TRACE's basic architecture, but suggest qualitative changes and additions to accommodate different languages, for instance.

(e) Examples of TRACE's operation; e.g. example of cascading processing in TRACE; example of TRACE discriminating between two similar words. TRACE is referred to in the context of the broader discussion of particular algorithms or architectural paradigms.

(v) Comparisons with other models. This category includes references to Shortlist (28) MERGE (12) Cohort (6) flmp (3) DCM (2) PARSYN (2) and a 
Changes over time, in the way in which TRACE's properties are referred to

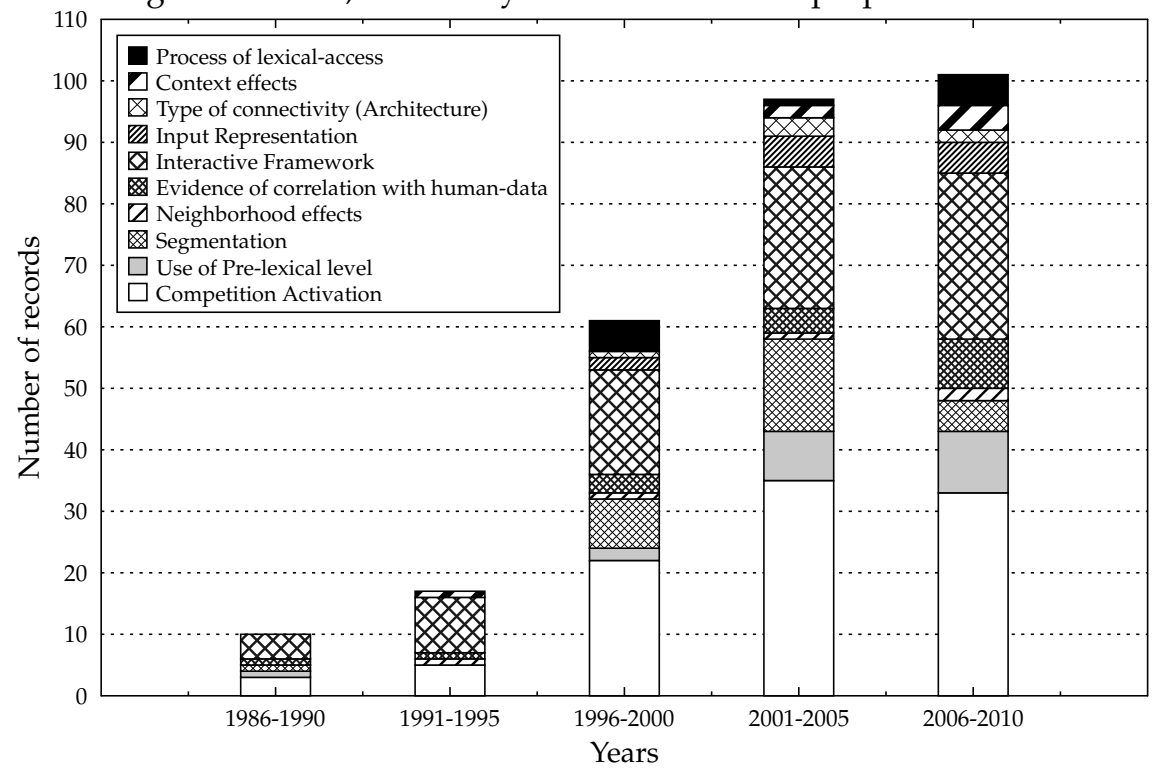

Fig. 5. The ways in which TRACE is referred to, as they change over time.

number of unnamed models, all forming a class of modern computational models of speech recognition. Most of the papers compare TRACE and one or more other models in a variety of contexts, most commonly with respect to cognitive plausibility. For example, Scharenborg and Boves ${ }^{18}$ compare and evaluate all the models with respect to the modularity versus interactivity debate. Fig. 6 shows how TRACE is cited in connection with the different models. Shortlist and MERGE generate most publications of the implemented models; the (non-implemented) Cohort Model generates the second highest number of publications. The theory of speech segmentation used in both Shortlist and MERGE was more differentiated than that of TRACE, and prompted comparison with TRACE. Overall, the data show the productivity of implemented models, with their behaviours being compared with human data.

Finally, in our corpus a special status exists for some 20 publications (part-) authored by McClelland, and counted among the categories analysed above.

\section{Discussion and Conclusions}

The data we have presented constitute perhaps the most detailed extant profile of the real research generated by a particular computational cognitive model. 


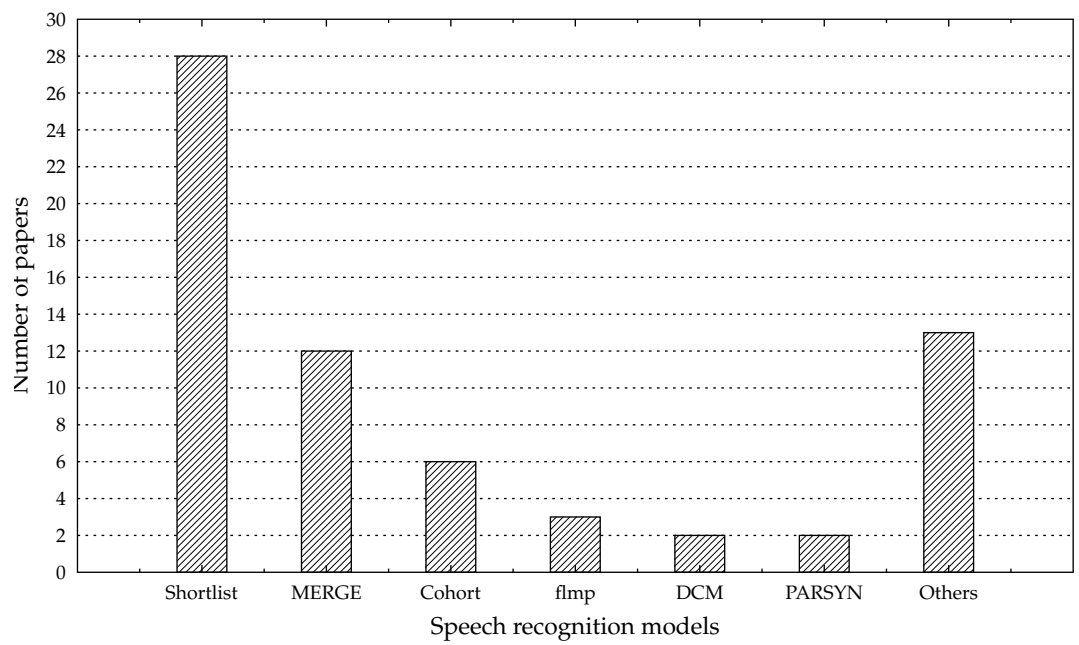

Fig. 6. Comparison of TRACE with other models.

Our analyses have shown that the TRACE model, as presented in the original 1986 paper, has had a developing "research life" of citations within wider discussions of speech perception, of simulations, of contrasts with newly proposed models, and of comparisons with other models and theories. Because it has been such a successful computational cognitive model, and because in many ways it typifies such modelling, conclusions drawn about TRACE have general validity across much of computational cognitive modelling. Some of these conclusions will seem uncontroversial from a conventional perspective, but they are important when we consider the philosophical status of such modelling.

First, researchers do not typically propose a model based on an explicit philosophical case for the assumptions implicit in the model. McClelland ${ }^{17}$ has subsequently reflected on the role of modelling in cognitive science, citing TRACE, but TRACE's hierarchical structure and its constituents were inherited from mainstream formal and laboratory linguistics.

Second, researchers do not tend to develop a particular computational cognitive model continually and incrementally, so as to progressively advance the understanding of the domain. TRACE was introduced and modified somewhat just three years later, but its basic architecture has remained the same, despite some quantitative manipulations of the parameters, for instance, and the size of the lexicon. As with TRACE, models may be explored with respect to more behavioural phenomena, or with respect to developments in mathematics, but the model's basic architecture typically remains unchanged along with the qualitative extent of the domain to which it refers. 
Third, modellers value simpler models over more complex ones. A model comprising only a few dimensions and some emergent behaviour(s) is seen as an end goal, as opposed to a step in the direction of real-world completeness. A theoretical perspective on complexity and/or completeness is currently a much smaller and a separate enterprise in cognitive science ${ }^{18}$. Missing from both approaches is a focus on identifying the essence of the processing domain, and subsequent exploration of how the modeller can move back and forth between this simple essence and the complexity of the complete real-world domain ${ }^{19}$.

Fourth, modellers frequently idealize model comparison as a contest against a set of benchmark data, the clear result of which will advance the field by leaving only one surviving model. Our analysis has shown a more complex picture in which a relatively small number of researchers typically make very partial comparisons between often just two models. Even then, the outcomes of the comparisons typically have to be judged against the differing strengths of the competing models and against the changing research interests of the modelling community (Fig. 5); the comparisons tend to be jousts not mortal combats. A major factor in model comparison is the availability of implemented versions of models; TRACE compares favourably, in this respect, with many models.

When we look at the entities that constitute a cognitive model, we typically find they are abstractions of a particular kind - in TRACE they are features, phonemes and words. Although our first impression is that these are "real" material entities, they are in fact abstract generalizations representing that which is taken to be similar across many actual instances (consider the phoneme $/ \mathrm{b} /$, for instance). They are universals, in this sense-abstract universals ${ }^{19}$.

Abstract universals allow us to investigate the ordered relations between such universals, as in TRACE's levels. In this sense, computational cognitive modelling resembles the study of formal syntax, which has always been cognitive science's core research paradigm.

Such ordered relations can be quantified, as in TRACE, and compared with human behaviours. However, associating numerical measures of activation with particular entities in a model does not change the abstract nature of the entities.

A model constituted solely from such universals is intrinsically always defeasible by real-world data. Prosodic information has never been incorporated into TRACE, and so the model is (trivially) "defeated" by such data from the real-world domain of speech perception. Similarly, TRACE's authors originally took no account of attention. Mirman et al. ${ }^{7}$ modelled certain effects of attention with respect to the abstract-universal entities of the original architecture, but such modelling can in turn be defeated by any of the long-known anatomical details of attentional processing, which were never in Mirman et al.'s sights. These "defeats" are not recognized as such by most researchers because the goal 
of most modelling is seen as an explanation couched in the simplest (preferably mathematical) abstract terms; the goal is not to connect with real-world complexity.

None of these points negates the fact that research with TRACE has provided researchers with an insightful conceptual vocabulary that has been reflected in interesting laboratory experiments on human speech perception. However, these points do raise the question, is there an alternative, or complementary, approach that has as its modelling goal an ever closer approximation to the completeness of the relevant domain, in all its complexity?

The suggestion made by Shillcock $(2014)^{19}$ is for (computational) cognitive models to be based around a different sort of universal (with its own philosophical history) - a concrete universal. Such a universal is "concrete" by virtue of being associated with everything else in the domain. It is also a particular of the domain, something material, something specific to the processing being studied. For speech perception, such a universal might be the sound associated with the vowel schwa. A representation of this sound in a model of speech processing is (a) capable of being enriched to capture its full variability as it occurs in real speech, (b) cannot be defeated by new real-world data or wished away by theorizing, and, critically, (c) occupies the position of a "hub" in the model, such that it connects with every other entity in the domain in an architecture very different from traditional hierarchical modelling. The identification of the schwa sound as the relevant concrete universal is an acknowledgement of its wide-ranging participation in speech phenomena, developmentally and in adult perception and production. This suggestion permits the qualitative development of conventional computational cognitive modelling, of which TRACE has been such a key example.

\section{References}

1. Chawla, M. (2011). A Theoretical Anatomy of a Successful Cognitive Model. Unpublished MSc dissertation, The University of Edinburgh.

2. Frigg, Roman and Hartmann, Stephan, "Models in Science", The Stanford Encyclopedia of Philosophy (Fall 2012 Edition), Edward N. Zalta (ed.), $\mathrm{URL}=<$ http://plato.stanford.edu/archives/fall2012/entries/modelsscience/>.

3. McClelland, J. L., \& Rumelhart, D. E. (1981). An interactive activation model of context effects in letter perception: I. An account of basic findings. Psychological Review, 88(5), 375-407.

4. Rumelhart, D. E., \& McClelland, J. L. (1982). An interactive activation model of context effects in letter perception: II. The contextual 
enhancement effect and some tests and extensions of the model. Psychological Review, 89(1), 60-84.

5. McClelland, J.L., \& Elman, J.L. (1986). The TRACE model of speech perception. Cognitive Psychology, 18, 1-86

6. Strauss, T. J., Harris, H. D., \& Magnuson, J. S. (2007). jTRACE: A reimplementation and extension of the TRACE model of speech perception and spoken word recognition. Behavior Research Methods, 39(1), 19-30.

7. Mirman, D., McClelland, J. L., Holt, L. L., \& Magnuson, J. S. (2008). Effects of attention on the strength of lexical influences on speech perception: Behavioral experiments and computational mechanisms. Cognitive Science, 32(2), 398-417.

8. Norris, D. (1994). Shortlist: A connectionist model of continuous speech recognition. Cognition, 52(3), 189-234.

9. Perruchet, P., \& Vinter, A. (1998). PARSER: A model for word segmentation. Journal of Memory and Language, 39(2), 246-263.

10. Luce, P.A. \& Pisoni, D.B. (1998). Recognizing spoken words: The neighborhood activation model. Ear and Hearing, 19(1), 1-36.

11. Luce PA, Goldinger SD, Auer ET and Vitevitch MS (2000). Phonetic priming, neighborhood activation, and PARSYN. Perception \& Psychophysics, 62, 615-25.

12. Gaskell, M., \& Marslen-Wilson, W. (1997). Integrating form and meaning: A distributed model of speech perception. Language and Cognitive Processes, 12(5-6), 613-656.

13. Norris, D., McQueen, J., \& Cutler, A. (2000). Merging information in speech recognition: Feedback is never necessary. Behavioral and Brain Sciences, 23(03), 299-325.

14. Norris, D., \& McQueen, J. (2008). Shortlist b: A bayesian model of continuous speech recognition. Psychological Review, 115(2), 357-395.

15. Massaro, D. W. (1998). Perceiving talking faces: From speech perception to a behavioral principle. Cambridge, MA: MIT Press.

16. Scharenborg, O. \& Boves, L. (2010). Computational modelling of spoken word recognition processes: Design choices and evaluation. Pragmatics \& Cognition, 18, 136-164.

17. McClelland, J. L. (2009). The place of modeling in cognitive science. Topics in Cognitive Science, 1(1), 11-38.

18. Van Orden G, Stephen DG (2012) Is cognitive science usefully cast as complexity science? Topics in Cognitive Science 4:3-6.

19. Shillcock, R. (2014). The concrete universal and cognitive science. Axiomathes 24, 63-80. 